\title{
ÜBER DAS ARCHIV DES HANSISCHEN GESCHICHTSVEREINS
}

\author{
von Antjekathrin Graßmann
}

Ende Februar 2009 konnten die archivischen Ordnungs- und Verzeichnungsarbeiten am Archivbestand des Hansischen Geschichtsvereins' beendet werden, der als Depositum ${ }^{2}$ dem Archiv der Hansestadt Lübeck anvertraut ist. Er unterliegt damit der hier geltenden Archivbenutzungsordnung und ist zu den Archivöffnungszeiten im Lesesaal des Archivs einsehbar. Eine ähnliche Übereinkunft ${ }^{3}$ besteht seit 1971 auch für die $\mathrm{Bi}$ bliothek des Hansischen Geschichtsvereins, deren Bände nach einem gewissen verabredeten Zeitraum ins Eigentum der Bibliothek des Archivs übergehen.

Anders als die durch Geschäftsordnung geregelte Abgabepflicht für das Schriftgut städtischer Behörden an das zuständige kommunale Archiv, liegt es hinsichtlich der Unterlagen von Vereinen, Firmen und Familien im Belieben der Verantwortlichen, ihre Überlieferung einem öffentlichen Archiv anzuvertrauen und es damit vor Verlusten oder dem völligen Untergang zu schützen. Dieser Charakter eines Privatarchivs bedingt allerdings auch häufig eine gewisse Zufälligkeit und Unregelmäßigkeit in der Überlieferung des Materials. Vereinsarchive sind eben auch organisch erwachsen; häufig fördern der mehrfache Wandel der Funktionsträger des Vereins und dessen fehlende Verankerung an einem Ort auch nicht gerade die Kontinuität der Aktenüberlieferung. Zudem ist mancher Schriftführer und Redakteur wortreich, ein anderer lakonisch. Ohnehin nimmt der Papierausstoß in der zweiten Hälfte des 20. Jahrhunderts stark zu. Die heutige Zeit elektronischer Kommunikation wird wiederum noch nicht kalkulierbare Auswirkungen auf archivische Arbeit und Bewertung nach sich ziehen.

Da die Vorsitzenden ${ }^{4}$ des HGV gemäß Satzung aus Lübeck stammen müssen, ist das HGV-Archiv bis auf eine Ausnahme zwischen 1972 und 1975 ortsfest geblieben. ${ }^{5}$ Auch hat es nicht zu den Lübecker Archivbe-

\footnotetext{
' Findbuch des Archiv der Hansestadt (=AHL): Signatur 5.4-30.

${ }^{2}$ AHL, Archiv des HGV Nr. 15.

${ }^{3}$ Ebd. Nr. 13.

+ Siche Liste am Schluss des Beitrags.

${ }^{5}$ Damals wurde es kurzzeitig nach Bremen überführt (Archiv des HGV Nr. 14).
} 
ständen gehört, die 1942 kriegsbedingt auf das Gebiet der späteren sowjetisch besetzten Zone ausgelagert wurden. Andererseits führte die enge Symbiose von Archiv und Geschäftsführung im gleichen Hause dazu, dass vieles an ephemerem Schriftgut in die Ablage/Archiv des HGV gelangte. Daher musste bei der Ordnung und Verzeichnung manches aus der Zeit von 1975 bis 2000 kassiert werden. Dennoch hat das Archiv des HGV auch so noch einen Umfang von immerhin 15 Regalmetern und ist damit eines der größten Vereinsarchive im Archiv der Hansestadt Lübeck. Es zählt insgesamt etwa 750 Nummern.

Bekanntlich wurde der Hansische Geschichtsverein 1871 gegründet, ${ }^{6}$ um die Sammlung und Veröffentlichung von Quellen zu fördern sowie der hansischen Geschichtsforschung einen Mittel- und Vereinigungspunkt zu geben. Seine Mitglieder kamen aus allen Teilen des Deutschen Reiches und seine Mitgliedsstädte vereinten die Kommunen von Elbing bis Köln, von Kiel bis Hannoversch-Münden.

Trotz der Entstehung und Verfestigung der Grenze zwischen den beiden deutschen Staaten nach dem 2. Weltkrieg seit 1949 blieb der Hansische Geschichtsverein noch lange Zeit einer der wenigen gesamtdeutschen Vereine. Die Verbindung zwischen Mitgliedern westlich und östlich des Eisernen Vorhangs konnte gewahrt werden. 1955 bildete sich eine Arbeitsgemeinschaft des Hansischen Geschichtsvereins in der Deutschen Demokratischen Republik, ${ }^{7}$ die, wie der Name ausdrückt, den persönlichen und wissenschaftlichen Kontakt zwischen den Mitgliedern beider deutschen Staaten weiterhin, wenn auch etwas eingeschränkt, gestattete. Zwar gab sich die Arbeitsgemeinschaft des HGV in der DDR 1969 eine eigene Satzung, aber zur endgültigen Spaltung des Vereins kam es erst im darauffolgenden Jahr, als staatliche Stellen der DDR die Trennung der Hansischen Arbeitsgemeinschaft vom $\mathrm{HGV}$ verfügten. ${ }^{8}$ Sie wurde erst mit der Wiedervereinigung Deutschlands 1989 beendet. 1990/1991 kam es zu einer Verschmelzung, die sich mit der Aufnahme ostdeutscher Mitglieder in den Vorstand des HGV und die Fortsetzung der Veröffentlichungsreihe „Hansische Studien“9 manifestierte. Zu den Jahresversammlungen des

\footnotetext{
${ }^{6}$ Ahasver v. Brandt, Hundert Jahre Hansischer Geschichtsverein. Ein Stück Sozial- und Wissenschaftsgeschichte, in: HGbll. 88/I (1970), S. 5-67.

${ }^{7}$ Prof. Dr. Eckhard Müller-Mertens (Berlin), ihr langjähriger Vorsitzender, arbeitet gegenwärtig an einer ausführlichen Darstellung und Würdigung der Arbeit der Arbeitsgemeinschaft, die in absehbarer Zeit in den HGbll. erscheinen wird.

${ }^{8}$ Dokumentation zur Entwicklung des Verhältnisses zur ehemaligen Arbeitsgemeinschaft des Hansischen Geschichtsvereins in der DDR und dem Gesamtverein Mai 1969-Juni 1971, in: HGbll. 89 (1971), S. 266-273.

${ }^{9}$ Sie erschienen ursprünglich (bis 1969) in der Reihe „Abhandlungen zur Handels- und Sozialgeschichte", hg. im Auftrag des HGV, seit 1970 hg. von der Hansischen Arbeitsgemeinschaft der Deutschen Historikergesellschaft (in der DDR).
} 
HGV, die übrigens auch bis 1970 zeitweilig noch in der ehemaligen DDR stattgefunden hatten, kam man seitdem auch wieder in ostdeutschen Hansestädten zusammen. Die Versammlungen sind wie eh und je ein Höhepunkt des Vercinslebens geblieben. ${ }^{10}$

us dem Gesagten ergibt sich, dass Unterlagen über die Verbindung des HGV und der Arbeitsgemeinschaft in der DDR im Hansischen Geschichtsvereins-Archiv bis in die 1960er Jahre vorhanden sind (10 Nummern), sich aber verständlicherweise in der DDR ein eigenes Archiv bildete. Die Unterlagen liegen in der Obhut des Universitätsarchivs der Humboldt-Universität Berlin und sind noch bis 2010 für die Benutzung gesperrt."

Was befindet sich nun im Archiv des Hansischen Geschichtsvereins? Der Bestand enthält das bei der Geschäftsstelle des HGV entstandene Archivgut, dem zum Teil Aktenablieferungen einzelner Vorstandsmitglieder, des Schatzmeisters und der Redakteure der Hansischen Geschichtsblätter einverleibt worden sind. Der Bestand wurde zuerst 1955 von Olof Ahlers geordnet und verzeichnet. Das seitdem in den vergangenen 50 Jahren angesammelte Schriftgut wurde 2008/09 von der Verfasserin archivisch bearbeitet und der Gesamtbestand in eine einheitliche Ordnung gebracht. Eine Konkordanz ermöglicht den Rückgriff auf die Signaturen schon vor den Ordnungsarbeiten 2009 benutzter HGV-Unterlagen, was freilich selten geschehen ist.

Vorstandsprotokolle von 1873 bis zur Gegenwart sind vorhanden, außer in den beiden Kriegszeiten, als das Vereinsleben ruhte. Die dem Verzeichnis zu entnehmenden zeitlichen Überlappungen erklären sich durch verschiedene, sich ergänzende, aber nicht jeweils vollständige Reihen, z. T. gebundener Bände, meist Ablieferungen von Vorstandsmitgliedern. Die Akten betreffend Vorstandsmitglieder ${ }^{12}$ erscheinen auf den ersten Blick vielversprechend, sind aber meistenteils recht dürftig (ca. 60 Nummern). Das Rechnungswesen wird durch Jahresrechnungen, Aufstellungen über Ein- und Ausgaben sowie durch Zuschusseinwerbungen (einige Beispiele), Haushaltsvoranschläge, Rechnungen der Verlage und Stipendien repräsentiert.

Seit den Anfängen des Hansischen Geschichtsvereins waren einstige Hansestädte als korporative Mitglieder sehr erwünscht. Deren Überlieferung ist dicht und reichlich und spiegelt zugleich die Umrisse des Deut-

\footnotetext{
${ }^{10}$ Hugo Weczerka, Die Tagungen des HGV 1971-1969 (mit Karte), in: HGbll. 88/I (1970), S. 68-71.

"Vertrag zwischen der Arbeitsgemeinschaft und der Humboldt-Universität: AHL. HGVArchiv Nr. 16.

${ }^{12}$ Hugo Weczerka, Die Vorstandsmitglieder des HGV 1871-1969, in: HGbll. 88/I (1970), S. $72-80$.
} 
schen Reiches in seiner territorialen Entwicklung scit 1871 wider (ca. 100 Nummern). Auch Mitgliederlisten seit 1881 sowie Unterlagen zur Mitgliederbewegung könnten nach archivarischem Verständnis von Interesse sein und wurden daher aufbewahrt (ca. 30 Nummern). Korrespondenzen mit Mitgliedern, aber auch mit Nichtmitgliedern wurden erhalten, darunter - vielleicht erwähnenswert - eine Umfrage von Ahasver von Brandt aus dem Jahr 1955 nach dem Stand hansischer Stadtarchive nach dem Kriege. ${ }^{13}$ Protokolle der Jahresmitgliederversammlungen liegen außer in einigen frühen Einzelstücken seit 1947 bis heute in einer vollständigen Reihe vor.

Großen Umfang ${ }^{14}$ nehmen dagegen die Unterlagen der Jahresmitgliederversammlungen ein, die mit Ausnahme der Kriegszeiten jährlich in den verschiedenen Hansestädten stattfanden und -finden. Auch hier tritt die Weitgespanntheit der historischen Hanse vor Augen, wenn man die umfangreichen Unterlagen, z. B. der Hansetagungen von Danzig und Breslau, von Dortmund und Köln, von Schleswig (ebenso wie Emden und Celle keine Hansestadt, aber trotzdem Ziel von Hansetagungen), von Stendal, Mühlhausen, Lemgo, Magdeburg, aber auch Zutphen und Zwolle in den Blick nimmt (ca. 100 Nummern). Auch Sonderveranstaltungen wie Fahrten nach Visby, nach Flandern und ins Baltikum wie auch Kolloquien in London, Visby, Brügge, Novgorod, Bergen, Amsterdam und King's Lynn findet man in den Akten repräsentiert.

Jahresberichte sind leider nur lückenhaft überliefert, finden sich aber oft in den „Hansischen Geschichtsblättern" abgedruckt. Breiten Raum nehmen die Briefwechsel und Verträge mit den Verlagen des Hansischen Geschichtsvereins ein, wogegen die Unterlagen über einzelne Veröffentlichungen höchst unterschiedlich und mehr zufällig vorhanden sind. Relativ dicht ist die Überlieferung zu den älteren Veröffentlichungen bis in die 1940er Jahre sowie - das ist eine Ausnahme - die aktenmäßige Darstellung des Projektes der „Hansischen Handelsstraßen“ (9 Nummern). Reichlich vorhanden sind Unterlagen der Redaktion der „Hansischen Geschichtsblätter" (Redakteure Johansen, Weczerka, Haase; über 50 Nummern), ebenso die Unterlagen zur Veröffentlichung der „Pfïngstblätter“ und der „Hansischen Volkshefte". Verschiedentlich fïndet man Unterlagen zu nicht realisierten Projekten, wie auch über die Teilnahme an fremden wissenschaftlichen Unternehmungen.

Den Schluss des Verzeichnisses bilden Manuskripte und Materialien im Rahmen des Hansischen Geschichtsvereins erschienener Veröffentlichun-

\footnotetext{
${ }^{1.3}$ AHL, HGV-Archiv Nr. 383.

${ }^{14}$ Hierin auch einige der sehr seltenen Fotos.
} 
gen (hauptsächlich zum Hansischen Urkundenbuch) sowie Schriftgut über den Tauschverkehr des Vereins (aufgrund der „Hansischen Geschichtsblätter") und über den Schriftenbestand.

Die Nachlassbestände der Historiker und Juristen Ludwig Beutin, Ferdinand Frensdorff, Anton Hagedorn, Konstantin Höhlbaum, Adolf Hofmeister, Paul Johansen, Karl Kunze, Klaus Nordmann, Fritz Rörig und Goswin von der Ropp sind jetzt in den Lübecker Archivbeständen „Nachlässe und Familienarchive" zu finden. Die Aufzeichnungen von Friedrich Bruns und Gerhard Schneider wurden ihren schon ohnehin im Archiv der Hansestadt Lübeck verwalteten Nachlassbeständen zugeordnet.

Hat man das gesamte Material durchgesehen, so entsteht vor allem ein farbiges Bild dieses Geschichtsvereins, der mehr die Qualität einer historischen Kommission denn eines Geschichtsvereins im eigentlichen Sinne besitzt. Seine Überregionalität, ja seine Internationalität - dieses seine besonderen Kennzeichen - werden augenfällig, ebenso seine Prägung durch die verschiedenen Vorstandsmitglieder, die ganz individuell geschichtliche, regionalgeschichtliche, rechtsgeschichtliche, aber auch kulturgeschichtliche Interessen vertraten und betonten. Über normale Geschichtsvereine hebt ihn auch sein deutsch-deutsches Schicksal hinaus. Überdies steht er selbst im Spannungsfeld der Beurteilung der Hanse und ihrer Geschichte, von der wir wissen, dass sie für verschiedene politische Auslegungen ja gar zu leicht verfügbar ist.

Manches Interessante ist zu finden, aber andererseits darf man wiederum nicht zuviel erwarten. Ein Archivbestand steht nicht allein, seine Informationen können ergänzt und flankiert werden durch die Einsichtnahme in andere Bestände des Archivs der Hansestadt Lübeck, z. B. den Nachlass Rörig, die schon erwähnten Nachlässe von Bruns und Schneider. Vielleicht lohnt auch ein Blick in die Nachlässe Emil Helms und Johannes Kretzschmar. Hier und da bieten die allgemeinen Archivakten, wie das Neue Senatsarchiv, die Unterlagen des Amtes für Kultur und des Polizeiamts ergänzende Informationen. Zudem, das darf nie vergessen werden, sind die „Hansischen Geschichtsblätter“" selbst eine sehr informative Quelle zur Vereinsgeschichte. So findet man dort auch authentische und konkrete Äußerungen aus der Sicht des Vereins selbst.

Aber erst in der Gegenwart werden die Geschichtsvereine stärker zu einem historischen Forschungsthema, indem sie in ihren zeitgenössischen Kontext eingeordnet werden und durch Vergleiche eine Würdigung des Phänomens „Geschichtsverein" versucht werden soll. ${ }^{15}$ Hierfür sind nun

\footnotetext{
${ }^{15}$ Ein wegen seiner methodischen Einseitigkeit nicht gelungenes Beispiel stellt der Beitrag von Helmut Stubbe da Luz dar, ..Die Arbeit in der gewohnten Form fortgesetzt"“? Der Verein
} 
durch die Ordnung und Verzeichnung der Unterlagen alle Möglichkeiten für die Forschung eröffnet.

Liste der Vorsitzenden

1871-1879 Stadtbibliothekar Wilhelm Mantels

1879-1903 Bürgermeister Wilhelm Brehmer

1903-1919 Bürgermeister Emil Ferdinand Fehling

1919-1928 Bürgermeister Johann Martin Neumann

1928-1934 Staatsarchivar Johannes Kretzschmar

1935-1950 Senator Georg Kalkbrenner

1950-1959 Stadtdirektor Emil Helms

1960-1975 Senator Gerhard Schneider

1976-1985 Bürgermeister Robert Knüppel

1986-1996 Senator Heinz Lund

1997-2010 Archivdirektorin Antjekathrin Graßmann

für Lübeckische Geschichte und Altertumskunde, die Bremer Historische Gesellschaft und

der Hansische Geschichtsverein in der NS-Zeit, in: Blätter für deutsche Landesgeschichte 141/142 (2006/2007), S. 289-345, hier: S. 330-345. 